\title{
Bridge Inspection and Reinforcement Technology Application
}

\author{
Hezhi Li and Fanghua Liu \\ Nanchang Key Laboratory of material and structure detection Jiangxi University of Technology
}

Keywords: Bridge; Non-destructive test; Load test; Reinforcement

\begin{abstract}
Most of the bridges built in 1950s and 1960s and even in 1970s have been beyond service period and have a lot of damages and defects, which are difficult to adapt to the rapid development of transportation today. If all these bridges are dismantled and rebuilt, it will require considerable cost, while some bridges also have historical and political significance. Thus, bridge inspection and reinforcement technology application is particularly important. Firstly, the article describes the regular examination contents for bridge and the commonly used conventional detection techniques in detail (non-destructive test method, semi-destructive test method, integrated use of semi-destructive test and non-destructive test method and load test) and gives examples of bridge inspection. Then, the article sums up the commonly used traditional bridge reinforcement methods today, and introduces the new composite material- carbon fiber polymer (referred to as CFRP) applied in bridge reinforcement in recent years and the methods of strengthening old bridges. Finally, the article introduces the comprehensive application of various methods of reinforcing bridge through examples of bridge reinforcement.
\end{abstract}

\section{Introduction}

With the development of the national economy, highway has also undergone change as a kind of quick transport. The completion of high-grade highways and bridges illustrates that human beings have entered a booming century. A bridge connecting roads plays a vital role. Cost for building highway bridges is very expensive, so people always take various measures to enable the project quality in the process of building the bridge. Nevertheless, highway bridge will be damaged in the natural environment (corrosion, temperature and humidity changes), and the operating environment (load effect and increasing frequency, material and structural fatigue), which is a non-reversal process. Bridges built in 1950s and 1960s and even in 1970s have been operating for 30-40 years, most have been beyond the service period and have a lot of damages and defects, which are difficult to adapt to the rapid development of transportation today. Therefore, old bridge maintenance and reinforcement is an important problem demanding prompt solution.

There are several major bridge accidents in our country. On September 24, 1998, the bridge fracture of Ningbo Bridge occurred in the upcoming closing procedure, and the loss has been up to 400 million yuan. On March 4, 1999, Chongqing Qijiang Rainbow Bridge collapsed and 40 people died, which has caused devastating social impact. On August 27, 2000, Taiwan's Kaoping Bridge deck fracture occurred, causing 16 cars plunged into the river, and 22 people were injured in the accident. On November 7, 2001, SichuanYibin South Gate bridge deck fracture collapsed.

At all times and in all over the world, there are many examples of bridge collapse, which are enough to give people a wake-up call. With the development of bridge construction, the formation and function of the bridge structure is becoming more complex, and the project scale becomes larger 
and larger, which have drawn people's attention to the modern bridge quality and life.

In order to avoid bridge collapse accident in the future, health monitoring and vibration control technology has undoubtedly become the main channel for disaster evolution research for the large bridge structure and the security assurance. Perfect bridge health monitoring system can verify the theory of bridge design and construction quality, and monitor local and global service status, structural damage and resistance fading and evolution law, identify structural damage and its location, a bridge safety and carry out durability assessment and forecasting and early warning of bridge accidents. If these bridge health inspection and vibration control systems are installed, which is equivalent to a "all-weather" Doctor for these bridges, and bridge construction and operation can be protected effectively.

Through comprehensive testing and comprehensive evaluation for bridges, different strengthening methods can be applied for different defects. There are no officially published norms for old bridge reinforcement at home and abroad. Therefore, this paper refers to a lot of books and papers about bridge inspection and reinforcement.

The article summarizes the common methods to strengthen the bridge, introduces the method of the newly developed "carbon fiber bridge reinforcement design" and lists the examples of the old bridge testing and strengthening to provide the necessary reference for future bridge inspection and reinforcement .

\section{Significance of bridge inspection and reinforcement technology research}

Since the 1970s, our country has embarked on the research for old bridge reinforcement technology. In the period of Sixth Five-Year Plan, extensive research and engineering practice have been carried out for the testing, evaluation and reinforcement methods of highway old bridge, which has achieved good social and economic benefits. In the period of Ninth Five-Year Plan, the Ministry of Transportation regards the "old bridge detection, evaluation, reinforcement technology application" as part of 1989-1990 Mastery Program in a timely manner, the Institute of Science and Technology Information is responsible for promotion.

Significance of bridge inspection and evaluation. The assessment for actual capacity of existing bridges will provide the necessary basis for the use and maintenance of the bridge.

(1) Various defects emerge in the main parts of bridges such as cracks, dislocation and so on due to the long hours of operation, the extent of damage and the actual carrying capacity can be determined through testing;

(2) Since extremely-large industrial equipment and container transportation is gradually increasing, overweight vehicles often cross bridges, whether the overweight vehicles can pass safely through the bridge can be determined through inspection and evaluation, and relevant technical information can be provided for a temporary reinforcement;

(3) The load rating for the existing bridge can be determined through inspection for the carrying capacity of original bridge in order to decide whether to increase its load rating by reinforcement;

(4) In case of severe disasters causing serious flaws on the bridge, the bridge needs to be evaluated and inspected to provide a reliable basis for reinforcement.

Establish and accumulate the necessary technical information to build bridge database.

(1) In case of incomplete bridge data or lack of information, technical information shall be re-established and accumulated through inspection; 
(2) Systematical collection of bridge technical data and the establishment of bridge data base are the basis of the bridge computer management system, which is designed to better manage and maintain bridges and guide future bridge maintenance, reinforcement and maintenance work.

Inspect the quality of the bridge structure to determine the reliability of the project, promote and e develop the old bridge assessment and the design and calculation theories of the new structures.

(1) The practicality and reliability of theory can be verified through inspection and evaluation for bridges with new structures to further identify problems and sum up lessons in order to improve and perfect design theory and structural style;

(2) Inspection and assessment for a number of important bridges or extremely-large bridge should be carried out after completion to assess the quality of its design and construction and determine the reliability of the project;

(3) Understand the actual stress state of the bridge to determine the safe carrying capacity of the structure and service conditions;

(4) The maintained and reinforced bridges should be inspected to examine the quality of maintenance and reinforcement and verify the reasonableness and reliability of reinforcement methods.

Reinforcement, transformation and utilization for old bridges are effective measures to maintain normal road traffic. Active introduction and development of the advanced technology, materials and equipment used for old bridge reinforcement and the determination of reasonable reinforcement and transformation programs can extend the service life of the old bridge and save money, and its significance and impact is far-reaching.

\section{Principles for bridge carrying capacity assessment}

Inadequate carrying capacity of the bridge can directly affect the service life of the bridge, it depends on many factors, including the following aspects:

Poor design - wrong calculation model, unreasonable structure design, inadequate experience, improper foundation and construction type.

bridge deck overloading - the design standards for the original bridges are lower, in fact, a large number of large-tonnage overloaded vehicles pass through the bridge, and the old road pavement repair also increases the dead load of the bridge deck, leading to the deck to be overload.

Improper construction - material does not meet the requirements, structure size is incorrect, the substrate is not in place to catch the time limit and so on.

Improper maintenance - expansion joints, bearings, drainage facilities, etc. haven't receive timely maintenance and cleaning, partial corrosion of steel or reinforcing steel bar hasn't been treated properly, the damaged bridge deck has not been repaired timely.

Erosion in the natural environment - erosion from gaseous, liquid, and solid hazardous substances, lower base is eroded and undermined and so on.

Corrosion and aging of materials - steel corrosion expansion, carbonation of concrete, stone weathering and so on

According to the Design Specifications for Highway Bridge and Culvert, the bearing capacity limit state and the normal using limit state of all bridge and culvert structures should be computed. The provision is not only the basic principle and criteria for the design and calculation for a new bridge, but also the basic principles and criteria for the old bridge carrying capacity assessment, strengthening and reform. 
Adaptive rate of the carrying capacity, adaptive rate of traffic capacity, adaptive rate of flood venting capacity and coincidence rate of the carrying capacity shall be applied to calculate for comprehensive assessment.

Adaptive rate of the bridge carrying capacity should be calculated as follows:

$$
\beta=\frac{m}{m_{0}} \times 100 \%
$$

Where: $\beta$ - Acceptability of Bridge carrying capacity;

- Total number of bridges in one route;

$\mathrm{m}$ - Total number of compliant bridges in one route;

Adaptive rate of traffic capacity should be calculated using the following formula:

$$
\beta_{1}=\frac{n}{N} \times 100 \%
$$

Where: - Adaptive rate of traffic capacity;

$\mathrm{n}$ - Total number of bridges required to meet the existing traffic in one route in computing traffic capacity;

$\mathrm{N}$ - Total number of bridges in one route.

Adaptive rate of flood venting capacity should be calculated by the formula:

$$
\beta_{k}=\frac{h}{H} \times 100 \%
$$

Where: $\beta_{k}$ - Adaptive rate of flood venting capacity;

$\mathrm{h}$ - Total number of bridges required to meet the flood venting in one route;

$\mathrm{H}$ - Total number of bridges in one route.

\section{Commonly used bridge inspection methods}

Due to the impact of various factors, there will be all kinds of damage during the service life of the bridge structure, it is necessary to conduct regular inspections (general inspection), periodic inspection (basic inspection), and special inspections (special inspection), which will play an important role in the bridge maintenance, early detection and timely repair.

Common methods for bridge inspection methods are: non-destructive test method, semi-destructive test method, integrated use of semi-destructive test and non-destructive test method and load test. Of which the first three are the detection of concrete strength, internal defects and the like. In general, non-destructive testing of concrete beams include integrity and strength testing, in recent years, no new method of nondestructive testing of concrete beams emerge or there is no new breakthrough, and the traditional rebound method and ultrasonic method are used for concrete strength inspection, while load test is a comprehensive inspection to detect the characteristics of the entire structure of the bridge and the carrying capacity.

Load tests include static load test and dynamic load test. Static load acts on a specified location on the bridge through the static load test, in order to be able to test out the static strain, static shift and crack of the structure, so as to infer the working condition and ability to use of the bridge structure under load. 
Objectives of bridge static load test are as follows. 1. Inspecting bridge design and construction quality;

2. Verifying that the bridge design theory and calculation methods;

3. Having a direct knowledge of the bridge structure bearing condition, in order to determine the actual carrying capacity of the bridge structure;

4. Accumulating scientific and technical information to enrich and develop the bridge calculation theory and construction technology.

Static load test is generally divided into three stages, namely: investigation of the bridge structure and design of testing program; loading test and observation phase; analysis of test results and summary and so on. As for the bridges that have received load tests, the bridge carrying capacity should be further determined based on the working conditions of experimental data analysis for the structure, to draw the identification conclusions for the new bridge acceptance or to be regarded as the basis for the identification and checking for the carrying capacity of old bridges, in addition, identification reports for the carrying capacity of bridges and the appraisal form for bridge bearing capacity can be prepared. General analysis and evaluation work include the assessment for the working conditions of structure, the strength and stability of the structure, foundation and basis, structure stiffness requirements, cracks, etc.

Objectives of dynamic load test are as follows. 1. Determining of dynamic properties of dynamic load, namely, value, direction, frequency and law of function and so on of the acting force is caused by the structure vibration;

2. Determining dynamic characteristic of the structure, such as natural frequency of vibration, damping force characteristics and natural mode of vibration and so on;

3. Determining the response of forced vibration of the structure under dynamic loads, such as amplitude, dynamic stress, impact factor and fatigue properties.

Three empty vehicles, a heavy vehicle, two heavy trucks and three heavy vehicles have been applied respectively to conduct the experiment, the measured value of the main arch deflection refers to Fig. 1

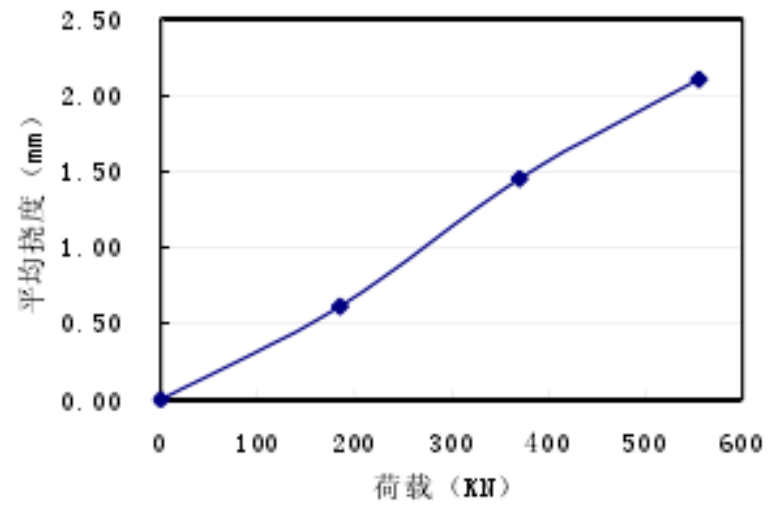

Fig. 1 Curve of arch deflection varied with load

When three empty vehicles, a load vehicle, two load vehicles and three load vehicles are applied respectively, the stress diagram refers to Fig. 2 


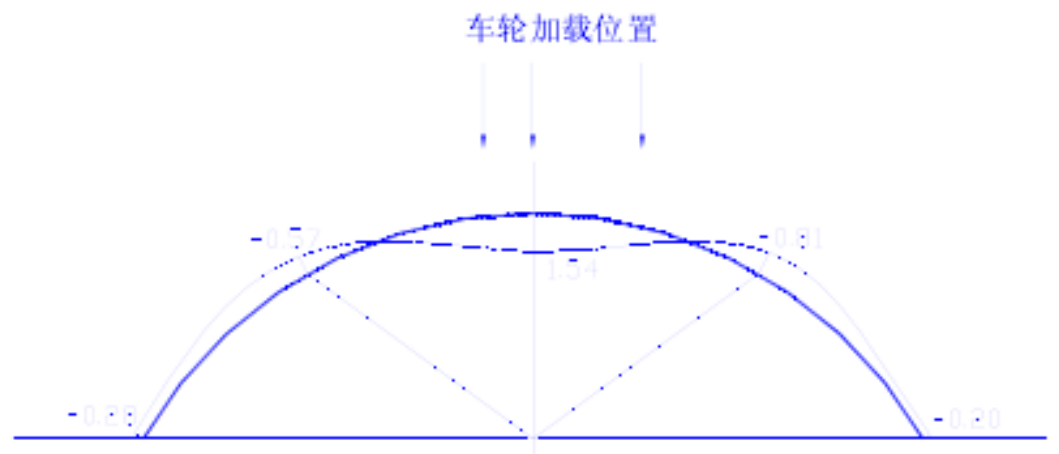

Fig. 2 Stress Diagram for three empty vehicles functioning on the bridge

\section{Bridge Reinforcement Technology Application}

The purpose for Reinforcement, renovation and transformation for the existing bridges is to apply existing resources in maximum and ensure their safe use and extend the service life, which is one of the major issues in international engineering area. At present, there are a number of aging bridges in our country, and the number of unsafe bridges is increasing year by year, therefore, the old bridge reinforcement has become imperative for bridge design, research and administration departments. Common methods for bridge reinforcement are the following:

Deck reinforcing layer reinforcement method. A layer of reinforced concrete is added on top of beam, and the old deck should be chiseled at first to ensure the old deck to be integrated with the main beam, in order to increase the effective height and compressive cross-sectional strength of the main beam and improve bridge transverse load distribution capabilities, so as to achieve the purpose of improving the carrying capacity of the bridge.

Outer wrapped concrete reinforcement method. Outer wrapped concrete reinforcement method is also known as cross section enlargement reinforcement method, in order to improve the strength, rigidity, stability and reduce the crack width, the cross-section and reinforcing bars are enlarged. For girder bridge, arch bridge, rigid frame bridges, piers, foundation and so on, the method can be applied if the conditions permit. Outer wrapped concrete will increase partial dead load of the original structure, therefore, in the formulation of the outer wrapped concrete size, whether the carrying capacity of outer wrapped member is enough or not should be taken into consideration, which is the premise of applying outer wrapped concrete program.

Steel plate paste reinforcement method. Due to increased traffic, insufficient carrying capacity of the main beam, or the severe corrosion in longitudinal reinforcement, or the serious transverse crack on the girder of the beam slab bridge, an adhesive, binder and anchor bolt will be used to paste and anchor the steel plate in the tension edge or weak parts of the concrete structure, so that the structure is formed integrally. Therefore, steel plate is used to replace reinforcing steel bars to improve the bearing capacity and durability of the bridge.

Ejector anchor concrete reinforcement method. At first, steel bar embedding method should be applied to embed the anchor bar into the structure that need to be reinforced, and then reinforcing steel mesh should be installed, concrete a certain thickness is ejected to form a composite structure together with the original structure of the force. Ejecting machine is used for ejector anchor concrete 
to eject the new concrete mixture into the surface of the anchored steel bars with jet nozzles and form a reinforced concrete after setting and hardening.

Reinforcement method of reducing the weight on the arch. Reducing the weight on the arch is a means to adjust the dead load distribution on the arch, although their purposes are all to restore and improve the carrying capacity of the original bridge, but their starting points and suitable situations are not the same. If the deformation of main arch ring is severe, dead load distribution on the arch should be adjusted to adjust the arch axis and pressure lines; if the basis carrying capacity of some two-way curved arch bridge is too weak, the weight of the building on the arch should be reduced to lower the requirements for the basis carrying capacity.

\section{Conclusions}

Based on the current situation and development trend of domestic and international bridges, the article raises awareness of the significance of detection and reinforcement technology research for bridge (old bridges built in the 1950s and 1960s in particular). On this basis, the article has described the common methods of bridge inspection and reinforcement in detail, at the same time, the article has also given a preliminary discussion about the new materials for bridge reinforcement - carbon fiber, calculation principle and construction technology.

In practical engineering application, a two-way curved arch bridge have been inspected by using the detection method proposed in the article - non-destructive test method and load test method, and the article also provide bridge inspection data and analysis results, and finally, the bridge testing work has been systematically summarized.

Bridge reinforcement has been a major issue in the engineering research at home and abroad, this paper has carried out summary for the traditional reinforcement methods and presented eight common reinforcement methods- Deck reinforcing layer reinforcement method, outer wrapped concrete reinforcement method, steel plate paste reinforcement method, ejector anchor concrete reinforcement method, reinforcement method of changing the structure force system, external pre-stressing reinforcement method, reinforcement method of reducing the weight on the arch and several reinforcement methods used for arch ring and arch rib.

\section{Acknowledgment}

This work was supported by Project on professional and characteristic construction of Jiangxi province 2010 (Civil Engineering) and Project on the planning and construction of disciplines in Jiangxi University of Technology (Structure Engineering)

\section{References}

[1] Ou Jinping Inspection and Control should be Strengthened for Large Bridges. China highway construction market, 4th Issue, 2004

[2] Liu Xiaoyao, Cai Jian, Liu Hui Damage Assessment of Bridge. Beijing: China Communications Press, May 2002.

[3] Yuan Wancheng, Cui Fei, Zhang Qiwei, Current Research and Development of Bridge Health Monitoring and Condition Assessment. Tongji University Journal, 1999.27(4). 
[4] Zhan Runshui, Hu Zhaofang, Shuai Changbin, Reinforcement Technology for Old Highway Bridge and Examples. Beijing: China Communications Press, 2001,10

[5] Xu Ben, Questions of Bridge Inspection and maintenance and Reinforcement Beijing: China Communications Press, 2002.3.

[6] Yi Gangxiang, Long Liaoqian, Xie Fang Summary of Bridge Reinforcement OVM communications, 2003.4

[7] Shao Xudong Questions of Bridge Design, Beijing: China Communications Press, 2003.3

[8] Standards of Ministry of Transportation of People's Republic of China. Design Code of Highway Reinforced Concrete and Prestressed Concrete Bridges and Culverts (JTJ023-85) Beijing: China Communications Press, 1985.

[9] Standards of Ministry of Transportation of People's Republic of China. Identification Methods for the Carrying Capacity of Old Highway Bridge. Beijing: China Communications Press,1990

[10] Industrial Standard of People's Republic. Highway Engineering Standard Beijing: China Communications Press, 2004.4 